\title{
Spectral Graph Attention Network with Fast Eigen-approximation
}

\author{
Heng Chang* \\ TBSI, Tsinghua University \\ changh17@mails.tsinghua.edu.cn \\ Wenbing Huang
Tsinghua University
hwenbing@126.com
}

\author{
Yu Rong \\ Tencent AI Lab \\ yu.rong@hotmail.com \\ Somayeh Sojoudi \\ University of California at Berkeley \\ sojoudi@berkeley.edu \\ Wenwu Zhu \\ Tsinghua University \\ wwzhu@tsinghua.edu.cn
}

\author{
Tingyang Xu \\ Tencent AI Lab \\ Tingyangxu@tencent.com \\ Junzhou Huang \\ University of Texas at Arlington \\ jzhuang@uta.edu
}

\begin{abstract}
Variants of Graph Neural Networks (GNNs) for representation learning have been proposed recently and achieved fruitful results in various fields. Among them, Graph Attention Network (GAT) first employs a self-attention strategy to learn attention weights for each edge in the spatial domain. However, learning the attentions over edges can only focus on the local information of graphs and greatly increases the computational costs. In this paper, we first introduce the attention mechanism in the spectral domain of graphs and present Spectral Graph Attention Network (SpGAT) that learns representations for different frequency components regarding weighted filters and graph wavelets bases. In this way, SpGAT can better capture global patterns of graphs in an efficient manner with much fewer learned parameters than that of GAT. Further, to reduce the computational cost of SpGAT brought by the eigendecomposition, we propose a fast approximation variant SpGATCheby. We thoroughly evaluate the performance of SpGAT and SpGAT-Cheby in semi-supervised node classification tasks and verify the effectiveness of the learned attentions in spectral domain.
\end{abstract}

\section{CCS CONCEPTS}

- Computing methodologies $\rightarrow$ Semi-supervised learning settings; Neural networks.

\section{KEYWORDS}

graph representation learning, graph spectral analysis, graph neural networks, deep learning

*Heng Chang is supported by 2020 Tencent Rhino-Bird Elite Training Program.

Permission to make digital or hard copies of all or part of this work for personal or classroom use is granted without fee provided that copies are not made or distributed for profit or commercial advantage and that copies bear this notice and the full citation on the first page. Copyrights for components of this work owned by others than ACM must be honored. Abstracting with credit is permitted. To copy otherwise, or republish, to post on servers or to redistribute to lists, requires prior specific permission and/or a fee. Request permissions from permissions@acm.org.

DLG-KDD '21, August 14-18, 2021, Virtual Conference

(c) 2021 Association for Computing Machinery.

ACM ISBN 978-1-4503-XXXX-X/18/06 _.\$15.00

https://doi.org/10.1145/1122445.1122456

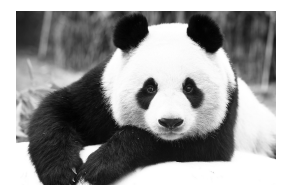

(a) Original image

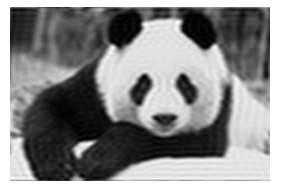

(b) Low-frequency (e.g., (c) High-frequency (e.g., background)

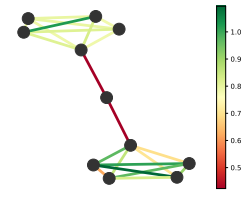

(e) Low-frequency outlines)

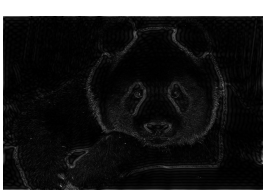

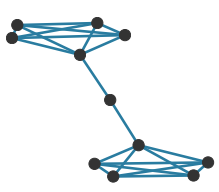

(d) Original graph

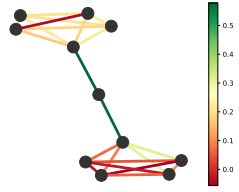

(f) High-frequency
Figure 1: Motivation: Separating the low- and highfrequency signals in both image and graph contributes to the feature learning. Color bars in graphs indicate the measurement of reconstructed edge weights.

ACM Reference Format:

Heng Chang, Yu Rong, Tingyang Xu, Wenbing Huang, Somayeh Sojoudi, Junzhou Huang, and Wenwu Zhu. 2021. Spectral Graph Attention Network with Fast Eigen-approximation. In DLG-KDD '21: The Sixth International Workshop on Deep Learning on Graphs: Methods and Applications, August 14-18, 2021, Virtual Conference. ACM, New York, NY, USA, 7 pages. https: //doi.org/10.1145/1122445.1122456

\section{INTRODUCTION}

Graph Neural Networks (GNNs) [39] aim at imitating the expressive capability of deep neural networks from grid-like data (e.g., images and sequences) to graph structures. The fruitful progress of GNNs in the past decade has made them a crucial kind of tools for a variety of applications, from social networks [13], computer vision [42], to chemistry [22].

Graph Attention Network (GAT) [35], as one central type of GNNs introduces the attention mechanism to further refine the convolution process in generic GCNs [19]. GAT, along with its variants $[11,36,37,43]$, considers the attention in a straightforward way: learning the edge attentions in the spatial domain. In this sense, this attention can capture the local structure of graphs, i.e., 
the information from neighbors. However, it is unable to explicitly encode the global structure of graphs. Furthermore, computing the attention weight for every edge in graphs is inefficient, especially for large graphs.

In computer vision, a natural image can be decomposed into a low spatial frequency component containing the smoothly changing structure, e.g., background, and a high spatial frequency component describing the rapidly changing fine details, e.g., outlines [7]. Figure 1(a) 1(c) depict the example of low- and high-frequency components on a panda image. Obviously, the contribution of different frequencies varies with respect to different downstream tasks.

Similar pattern can be observed more naturally in graphs. According to graph signal processing (GSP), we can directly divide the lowand high-frequency components based on the ascending ordered eigenvalues of Laplacian in graphs. The eigenvectors associated with small eigenvalues carry smoothly varying signals, encouraging neighbor nodes to share similar values (local information). In contrast, the eigenvectors associated with large eigenvalues carry sharply varying signals across edges (global information) [10, 25]. As demonstrated in Figure 1(d) 1(f), a barbell graph tends to retain the information inside the clusters when it is reconstructed with only low-frequency components(1(e)), but reserve knowledge between the clusters when constructed with only high-frequency ones (1(f)). Moreover, recent works [5, 17] also reveal the different contributions of low- and high-frequency components in graphs to the learning of modern GNNs.

In this paper, to model the importance of low- and high-frequency components in graphs, we propose to extend the attention mechanism to the spectral domain. In this way, we can explicitly encode the structural information of graphs from a global perspective. Accordingly, we present Spectral Graph Attention Network (SpGAT). In SpGAT, we choose the graph wavelets as the spectral bases and decompose them into low- and high-frequency components with respect to their indices. Then we construct two distinct convolutional kernels according to the low- and high-frequency components and apply the attention mechanism on both kernels to capture their importance respectively. Finally, an pooling function as well as an activation function are applied to produce the output. Figure 2 provides an overview of the design of SpGAT. Furthermore, we employ the Chebyshev polynomial approximation to compute the spectral wavelets of graphs and propose an variant SpGAT-Cheby, which is more efficient on large graphs. We thoroughly validate the performance of SpGAT and SpGAT-Cheby on five benchmarks with fourteen competitive baselines. SpGAT and SpGAT-Cheby achieve state-of-the-art results on all of the datasets.

\section{THE PROPOSED FRAMEWORK}

We denote $\mathcal{G}=(\mathcal{V}, \mathcal{E})$ as an undirected graph, where $|\mathcal{V}|=n$ is the set of $n$ nodes, and $\mathcal{E}$ is the set of edges, where $\left(v_{i}, v_{j}\right) \in \mathcal{E}$. The adjacency matrix is defined as $A \in \mathbb{R}^{n \times n}$, where $A_{i j}=1$ indicates an edge $\left(v_{i}, v_{j}\right)$. We denote $\boldsymbol{D}$ as the degree matrix. $\hat{A}=$ $\hat{D}^{-1 / 2}(A+I) \hat{D}^{-1 / 2}$ refers to the normalized adjacency matrix with self-loop, where $I$ is the identity matrix and $\hat{D}=D+I$.

From the spatial perspective, GNNs can usually be viewed as the feature aggregation among the neighbors of nodes in the spatial domain of graphs. Therefore, we write the feed-forward layer of

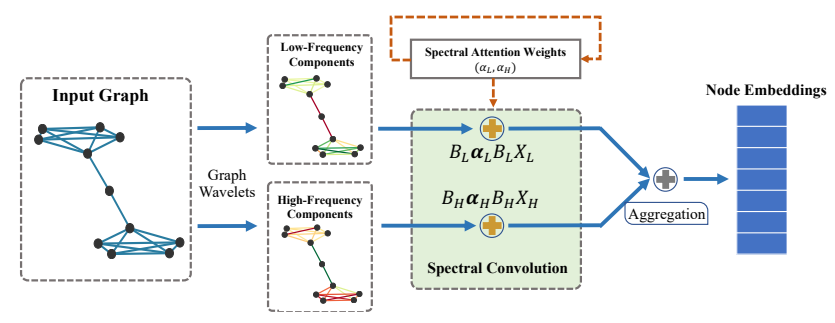

Figure 2: Overview of proposed Spectral Graph Attention Network(SpGAT). Two distinct convolutional kernels according to the low- and high-frequency components are constructed and the attention mechanism is employed on both kernels to capture the importance, respectively.

GNNs in a general form:

$$
\boldsymbol{h}_{i}^{\prime}=\sigma\left(\mathrm{AGG}_{j \in \mathcal{N}_{i}}\left(\alpha_{i j} \Theta \boldsymbol{h}_{j}\right)\right),
$$

where $\mathcal{N}_{i}$ refers to the neighborhood set of node $i$ in graph. $\boldsymbol{H}^{\prime}=$ $\left\{\boldsymbol{h}_{1}^{\prime \mathrm{T}}, \cdots, \boldsymbol{h}_{n}^{\prime \mathrm{T}}\right\}$ are the output of hidden vectors from the layer with $\boldsymbol{H}=\left\{\boldsymbol{h}_{1}^{\mathrm{T}}, \cdots, \boldsymbol{h}_{n}^{\mathrm{T}}\right\}$ as the input features. $\sigma(\cdot)$ refers to the activation function, such as ReLU. $\Theta^{\mathrm{T}} \in \mathbb{R}^{p \times q}$ refers to the learning parameters of the layer, where $p$ and $q$ refer to the feature dimensions of input and output, respectively. $\alpha_{i j}$ refers to the aggregation weight of neighbor $j$ for node $i$. AGG(.) refers to the aggregation function that aggregates the output of each neighbor, such as SUM and MEAN. For examples, VanillaGCN can be viewed as the special case of Eq. (1) where $\alpha_{i j}=\hat{A}_{i j}$, and AGG(.) $=\operatorname{SUM}(\cdot)$. Meanwhile, GAT proposes to compute the weight $\alpha_{i j}$ by a self-attention strategy and uses SUM as aggregation function.

Other than the neighbor aggregation in the spatial domain [19], VanillaGCN can also be understood from the perspective of GSP in the spectral domain:

$$
g_{\theta} \star x=B g_{\theta} B^{\mathrm{T}} \boldsymbol{x},
$$

where $\boldsymbol{x}$ is a signal on every node. $\boldsymbol{B}=\left\{\boldsymbol{b}_{1}, \cdots, \boldsymbol{b}_{n}\right\}$ are the spectral bases extracted from the graph. $g_{\theta}=\operatorname{diag}(\theta)$ is a diagonal filter parameterized by $\theta$. Given Eq. (2), VanillaGCN can be viewed as the spectral graph convolution based on the Fourier transformation on graphs with the first-order Chebyshev polynomial approximations [19]. Further, we can separate the spectral graph convolution into two stages [40]:

$$
\begin{aligned}
& \text { feature transformation : } \boldsymbol{X}=\boldsymbol{H} \Theta^{\mathrm{T}}, \\
& \text { graph convolution }: \boldsymbol{H}^{\prime}=\sigma\left(\boldsymbol{B} \boldsymbol{F} \boldsymbol{B}^{\mathrm{T}} \boldsymbol{X}\right) .
\end{aligned}
$$

In Eq. (3), $\boldsymbol{F}$ is a diagonal matrix for the kernel of graph convolution. For instance, the convolutional kernel for VanillaGCN is $\boldsymbol{F}=\operatorname{diag}\left(\lambda_{1}, \cdots, \lambda_{n}\right)$, where $\left\{\lambda_{i}\right\}_{i=1}^{n}$ are the eigenvalues of the normalized Laplacian $\boldsymbol{L}=\boldsymbol{I}-\hat{\boldsymbol{A}}$ in ascending order, while the spectral bases $\boldsymbol{B}$ for VanillaGCN are the corresponding eigenvectors.

\subsection{The Construction of SpGAT Layer}

In this section, we start to describe the construction of SpGAT layer. From the perspective of GSP, the diagonal values $\left(f_{1}, \cdots, f_{n}\right)$ on $F$ can be treated as the frequencies on graphs when they equal to the eigenvalues. We denote the diagonal values with small / large indices as the low / high frequencies, respectively. Meanwhile, 
the corresponding spectral bases in $B$ are low- and high-frequency components. As discussed in Section 1, the low- and high-frequency components carry different structural information in graphs. In this vein, we first propose to split the spectral bases into two groups and re-write Eq. (3) as follows:

$$
\begin{aligned}
\boldsymbol{X}_{\boldsymbol{L}} & =\boldsymbol{H} \Theta_{L}^{\mathrm{T}}, \boldsymbol{X}_{H}=\boldsymbol{H} \Theta_{H}^{\mathrm{T}}, \\
\boldsymbol{H}^{\prime} & =\sigma\left(\operatorname{AGG}\left(\boldsymbol{B}_{L} \boldsymbol{F}_{L} \boldsymbol{B}_{L}^{\mathrm{T}} \boldsymbol{X}_{L}, \boldsymbol{B}_{H} \boldsymbol{F}_{H} \boldsymbol{B}_{H}^{\mathrm{T}} \boldsymbol{X}_{H}\right)\right),
\end{aligned}
$$

where $\boldsymbol{B}_{L}=\left(\boldsymbol{b}_{1}, \cdots, \boldsymbol{b}_{d}\right)$ and $\boldsymbol{B}_{H}=\left(\boldsymbol{b}_{d+1}, \cdots, \boldsymbol{b}_{n}\right)$ are the lowand high-frequency components, respectively. Here $d$ is a hyperparameter that determines the splitting boundary of low- and highfrequency. When $\operatorname{AGG}(\cdot)=\operatorname{SUM}(\cdot)$, Eq. (4) is equivalent to the graph convolution stage in Eq. (3).

In Eq. (4), $F_{L}$ and $F_{H}$ can also be viewed as the importances of the low- and high-frequency. Therefore, we introduce the learnable attention weights by exploiting the re-parameterization trick:

$$
\boldsymbol{H}^{\prime}=\sigma\left(\operatorname{AGG}\left(\boldsymbol{B}_{L} \boldsymbol{\alpha}_{L} \boldsymbol{B}_{L}^{\mathrm{T}} \boldsymbol{X}_{L}, \boldsymbol{B}_{H} \boldsymbol{\alpha}_{H} \boldsymbol{B}_{H}^{\mathrm{T}} \boldsymbol{X}_{H}\right)\right) .
$$

In Eq. (5), $\boldsymbol{\alpha}_{L}=\operatorname{diag}\left(\alpha_{L}, \cdots, \alpha_{L}\right)$ and $\boldsymbol{\alpha}_{H}=\operatorname{diag}\left(\alpha_{H}, \cdots, \alpha_{H}\right)$ are parameterized by two learnable weights $\alpha_{L}$ and $\alpha_{H}$, respectively. To ensure $\alpha_{L}$ and $\alpha_{H}$ are positive and comparable, we normalize them by the softmax function in an attention manner:

$$
\alpha_{*}=\operatorname{softmax}\left(\alpha_{*}\right)=\frac{\exp \left(\alpha_{*}\right)}{\sum_{*} \exp \left(\alpha_{*}\right)}, \quad *=L, H .
$$

Theoretically, there are many approaches to re-parameterize $\boldsymbol{\alpha}_{L}$ and $\boldsymbol{\alpha}_{H}$, such as self-attention w.r.t the spectral basis $\boldsymbol{b}_{i}$. However, these kinds of re-parameterization can not reflect the nature of low- and high-frequency components. On the other hand, they may introduce too many additional learnable parameters, especially for large graphs. These parameters might prohibit the efficient training due to the limited amount of training data in graph learning, especially under the graph-based semi-supervised setting.

\subsection{Choice of Spectral Bases}

Another important design is the choice of the spectral basis. Instead of Fourier bases, we choose graph wavelets as spectral bases in SpGAT following the observation on the advantages of spectral wavelets in recent works [10, 40]. Formally, the wavelet on a graph $\psi_{s i}(\lambda)$ is defined as the signal resulting from the modulation in the spectral domain of a signal $x$ centered around the associated node $i[16,31]$. Then, given a graph $G$, the graph wavelet transformation is conducted by employing a set of wavelets $\Psi_{s}=$ $\left(\psi_{s 1}\left(\lambda_{1}\right), \psi_{s 2}\left(\lambda_{2}\right), \ldots, \psi_{s n}\left(\lambda_{n}\right)\right)$ as bases:

$$
\Psi_{s}(\lambda)=\boldsymbol{U} g_{s}(\lambda) U^{\mathrm{T}},
$$

where $\boldsymbol{U}$ is the eigenvectors of the normalized Laplacian $\boldsymbol{L}=$ $I-\hat{A} \cdot g_{s}(\lambda)=\operatorname{diag}\left(g_{s}\left(\lambda_{1}\right), g_{s}\left(\lambda_{2}\right), \ldots, g_{s}\left(\lambda_{n}\right)\right)$ is a scaling matrix with heat kernel scaled by hyperparameter $s$. The inverse of graph wavelets $\Psi_{s}^{-1}(\lambda)$ is obtained by simply replacing $g_{s}(\lambda)$ with $g_{s}(-\lambda)[10]$. Smaller indices in graph wavelets correspond to lowfrequency components and vice versa. Overall, the architecture of SpGAT layer with graph wavelet $\Psi_{S}$ as bases can be written as:

$$
X=H \Theta^{\mathrm{T}}, \quad H^{\prime}=\sigma\left(\operatorname{AGG}\left(\Psi_{s L} \alpha_{L} \Psi_{s L}^{-1} X, \Psi_{s H} \alpha_{H} \Psi_{s H}^{-1} X\right)\right) .
$$

\subsection{Parameter Complexity of SpGAT}

In Eq. (7), aiming to further reduce the parameter complexity, we share the parameters in feature transformation stage for $X_{L}$ and $X_{H}$, i.e., $\Theta_{L}=\Theta_{H}$. In this way, we reduce the parameter complexity from $O(2 \times(p \times q+1))$ to $O(p \times q+2)$, which is nearly the same as VanillaGCN, which is $O(p \times q)$. The parameter complexity of SpGAT is much less than that of GAT with $K$-head attention, which is $O((p+2) \times q \times K)$. Comparing with GAT, which captures the local structure of graphs from spatial domain, our proposed SpGAT could better tackle global information by combining the low- and high-frequency features explicitly from spectral domain.

\section{FAST APPROXIMATION OF SPGAT}

In SpGAT, directly computing the transformation according to Eq. (6) is intensive for large graphs, since diagonalizing Laplacian $L$ commonly requires $O\left(n^{3}\right)$ computational complexity. Fortunately, we can employ the Chebyshev polynomials to fast approximate the spectral graph wavelets without eigen-decomposition[16].

THEOREM 1. Let $s$ be the scaling parameter in the heat kernel $g_{s}(\lambda)=e^{-\lambda s}$, and $M$ be the degree of the Chebyshev polynomial approximation for the scaled wavelet (larger value of $M$ yields more accurate approximation but higher computational cost in the opposite), then the graph wavelet is given by

$$
\Psi_{s}(\lambda)=\frac{1}{2} c_{0, s}+\sum_{i=1}^{M} c_{i, s} T_{i}(\tilde{L}), c_{i, s}=2 e^{s} J_{i}(s),
$$

where $\tilde{\boldsymbol{L}}=\frac{2}{\lambda_{\max }} \boldsymbol{L}-\boldsymbol{I}, T_{i}(\tilde{\boldsymbol{L}})$ is the $i_{\text {th }}$ order Chebyshev polynomial, and $J_{i}(s)$ is the modified Bessel function of the first kind.

Theorem 1 can be derived from Section 6 in [16]. It should be noted that though [40] discusses the possibility to bring the method from [16] into approximating wavelets but with integral operations, we make the first attempt to integrate Theorem 1 into practice. Moreover, to accelerate the computation, we build a look-up table for the Bessel function $J_{i}(s)$ to avoid additional integral operations.

With this Chebyshev polynomial approximation, the computational cost of the spectral graph wavelet is decreased to $O(M\|\mathcal{E}\|+$ $M n$ ), where $\|\mathcal{E}\|$ is the total number of edges. Due to the real world graphs are usually sparse, this computational reduction can be very significant. We denote SpGAT with Chebyshev polynomial approximation as SpGAT-Cheby. As for SpGAT-Cheby, instead of using eigen-decomposition, we directly employ Eq.(8) to speed up the computation of the spectral wavelets $\Psi_{S}(\lambda)$. After that, the approximated $\Psi_{S}(\lambda)$ are seamlessly fed into the original SpGAT.

\section{EXPERIMENTS}

\subsection{Experimental Setup}

Joining the practice of previous works, we mainly focus on five node classification benchmarks under semi-supervised setting with different graph size, feature type and public splitting, including three citation networks: Citeseer, Cora and Pubmed [29], a coauthor network: Coauthor CS, and a co-purchase network: Amazon Photo [26]. Statistical overview of all datasets can be found in [30].

We thoroughly evaluate the performance of SpGAT with fourteen representative baselines: 


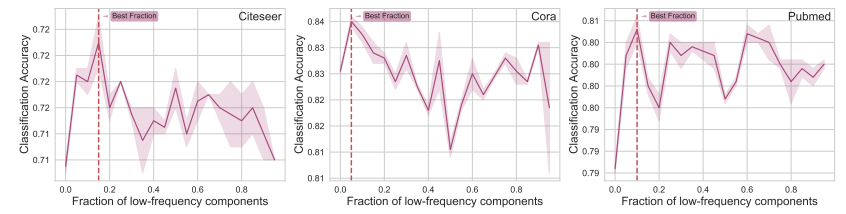

Figure 3: The performance of SpGAT w.r.t the proportion of low-frequency $d$ on citation datasets. The best fraction is marked with the dashed vertical line.

- Traditional graph embedding methods: DeepWalk [28] and Planetoid [41];

- Spectral-based GNNs: ChebyNet [8], VanillaGCN [19],SGC [38], GWNN [40], ARMA [3], and GZoom(DGI) [9];

- Spatial-based GNNs: GGNN [21], GraphSAGE [15], GAT [35], HyperGraph [2], HighOrder [27], and APPNP [20].

For all experiments, a 2-layer neural network is constructed using TensorFlow [1] with 64 hidden units. We train our model utilizing the Adam optimizer [18] with an initial learning rate $l r=0.01$. Early stopping is used with a window size of 100. Most training processes are stopped in less than 200 steps as expected. We initialize the weights following [12], employ $5 \times 10^{-4}$ L2 regularization and dropout the input and hidden layers to prevent overfitting [32]. For constructing wavelets, we set $s=1, t=1 \times 10^{-4}$ for SpGAT, and $M=1, s=2$ and $t=1 \times 10^{-4}$ for SpGAT-Cheby on all datasets. In addition, we employ the grid search to determine the best $d$ of low-frequency components and the impact of this parameter would be discussed in Section 4.3. Two variants with MEAN-pooling and MAX-pooling are implemented to demonstrate the effectiveness of aggregation function in SpGAT and SpGAT-Cheby. Without other specification, we use MAX-pooling in both models.

\subsection{Semi-supervised Node Classification}

Table 1 summaries the results on all datasets. For all baselines, we reuse the results from their public literature. From Table 1, we have these findings: (1) Clearly, the attention-based GNNs (GAT, SpGAT and SpGAT-Cheby) achieve relatively better performance across all datasets. It validates that the attention mechanism can capture the important patterns from either spatial or spectral perspective. (2) Specifically, SpGAT and SpGAT-Cheby achieve the best performance across all datasets. Particularly on Coauthor CS, the best accuracy by SpGAT-Cheby-MAX is $92.5 \%$ and it is better than the previous best (90.7\%), which is regarded as a remarkable boost considering the challenge on this benchmark. (3) Compared with MEAN aggregation, MAX aggregation seems to be a better choice for both models. This may due to that MAX aggregation can preserve the significant signals learned by SpGAT. (4) It is worthy to note that to achieve such results, both SpGAT and SpGAT-Cheby only employ the attention on low- and high-frequency of graphs in spectral domain, while GAT needs to learn the attention weights on every edge in spatial domain. It verifies that SpGAT is more efficient than GAT, since the global information of graphs can be better captured from spectral domain while with less parameters.

\subsection{Ablation Studies}

4.3.1 The Impact of Proportion of Low-frequency Components d. To evaluate the impact of the hyperparameter $d$, we fix the other
Table 1: Experimental results (in percentage) on semisupervised node classification.

\begin{tabular}{lccccc}
\hline Model & Citeseer & Cora & Pubmed & Coauthor CS & Amazon Photo \\
\hline DeepWalk [28] & 43.2 & 67.2 & 65.3 & - & - \\
Planetoid [41] & 64.7 & 75.7 & 77.2 & - & - \\
\hline ChebyNet [8] & 69.8 & 81.2 & 74.4 & 90.5 & 89.6 \\
VanillaGCN [19] & 70.3 & 81.5 & 79.0 & 89.8 & 90.6 \\
GWNN [40] & 71.7 & 82.8 & 79.1 & 90.3 & 88.5 \\
ARMA [3] & 70.9 & 83.3 & 78.4 & 90.6 & 86.4 \\
SGC [38] & 71.9 & 81.0 & 78.9 & 89.3 & 90.1 \\
GZoom(DGI) [9] & 71.7 & 83.2 & 77.1 & 88.9 & 89.3 \\
\hline GGNN [21] & 64.6 & 77.6 & 75.8 & 86.6 & 74.1 \\
GraphSAGE [15] & 67.2 & 74.5 & 76.8 & 90.1 & 90.1 \\
GAT [35] & 72.5 & 83.0 & 79.0 & 85.5 & 89.7 \\
HyperGraph [2] & 71.2 & 82.7 & 78.4 & 86.9 & 87.5 \\
HighOrder [27] & 64.2 & 76.6 & 75.0 & 84.2 & 26.1 \\
APPNP [20] & 72.7 & 83.1 & 79.1 & 90.7 & 91.8 \\
\hline \hline SpGAT-MEAN & $71.6 \pm 0.2$ & $82.6 \pm 0.3$ & $80.3 \pm 0.2$ & $91.0 \pm 0.3$ & $91.8 \pm 0.3$ \\
SpGAT-MAX & $72.1 \pm 0.2$ & $\mathbf{8 3 . 7} \pm 0.2$ & $\mathbf{8 0 . 6} \pm 0.3$ & $91.6 \pm 0.3$ & $91.4 \pm 0.2$ \\
SpGAT-Cheby-MEAN & $70.0 \pm 0.2$ & $80.7 \pm 0.4$ & $78.3 \pm 0.3$ & $91.1 \pm 0.2$ & $92.4 \pm 0.1$ \\
SpGAT-Cheby-MAX & $71.1 \pm 0.4$ & $82.1 \pm 0.3$ & $80.2 \pm 0.2$ & $\mathbf{9 2 . 1} \pm 0.1$ & $\mathbf{9 2 . 8} \pm 0.2$ \\
\hline
\end{tabular}

Table 2: Running time (s) comparison for obtaining spectral wavelets $\Psi_{s}(\lambda)$ between SpGAT and SpGAT-Cheby.

\begin{tabular}{ccc}
\hline Models & Eigen-decomposition & Fast approximation \\
\hline Citeseer & 11.23 & $5.19(\sim 2.2 \times)$ \\
\hline Cora & 5.79 & $2.78(\sim 2.1 \times)$ \\
\hline Pubmed & 1185.12 & $150.79(\sim 7.9 \times)$ \\
\hline
\end{tabular}

hyperparameters and vary $d$ from 0 to $100 \%$ linearly to run SpGAT on citation datasets. Figure 3 depicts the mean (in bold line) and variance (in light area) of every $d$. As shown in Figure 3, the mean value curve of three datasets exhibits similar pattern, that is, the best performance is achieved when $d$ is small. The best proportions of low-frequency components are $15 \%, 5 \%$ and $10 \%$ for Citeseer, Cora and Pubmed, respectively. In the other words, consistently, only a relatively small fraction of components needs to be treated as low-frequency components in SpGAT. This finding is consistent with the argument from [25], which has discussed that a small fraction of low-frequency components already contains sufficient information for the reconstruction of signals. Thus it might be a good choice to select a small $d$ for our model when generalizing to other datasets. A theoretically heuristic method to determine $d$ could be interesting and will be left for future exploration.

4.3.2 Time Efficiency of SpGAT and SpGAT-Cheby. As discussed in Section 3, we propose the fast approximation of spectral wavelets $\Psi_{s}(\lambda)$ according to Chebyshev polynomials. To elaborate its efficiency, we compare the time cost of calculating $\Psi_{s}(\lambda)$ between via eigen-decomposition (SpGAT) and fast approximation (SpGATCheby). We report the mean time cost of SpGAT and SpGATCheby with second-order Chebyshev polynomials after 10 runs for citation datasets. As shown in Table 2, we can find that this fast approximation can greatly accelerate the training process. Specifically, SpGAT-Cheby runs $7.9 \times$ times faster than SpGAT for obtaining $\Psi_{s}(\lambda)$ on the relatively large dataset Pubmed. It further validates the scalability of the fast approximation approach.

Meanwhile, we also compare the running time cost of 200 epochs among our proposed methods, VanillaGCN and GAT on Pubmed dataset, and the results can be found in Figure 4. We can observe that SpGAT runs slightly faster than GAT. Furthermore, with the fast approximation technique, SpGAT-Cheby saves nearly half of the time comparing with GAT and achieves comparable efficiency 


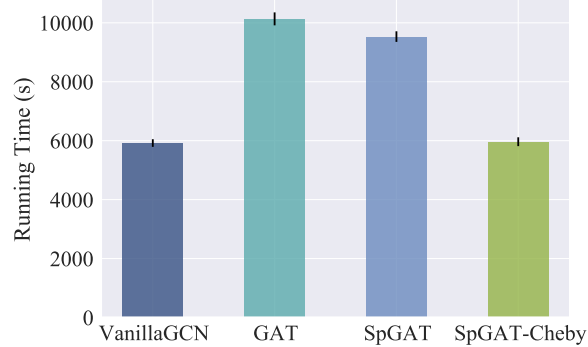

Figure 4: Running time $(s)$ comparison on Pubmed.

against VanillaGCN. It further confirms the efficiency of the proposed approximation on spectral wavelets.

\section{CONCLUSION}

In this paper, we propose SpGAT, a novel spectral-based graph convolutional neural network to learn the representation of graphs with respect to different frequency components in the spectral domain. By introducing the distinct trainable attention weights for low- and high-frequency components, SpGAT can effectively capture both local and global information in graphs and enhance the performance of GNNs. Furthermore, a fast variant SpGATCheby based on Chebyshev polynomial approximation is proposed to accelerate the spectral graph wavelets calculation and benefit the scalability. To the best of our knowledge, this is the first attempt to adopt the attention mechanism to the spectral domain of graphs. It is expected that SpGAT and SpGAT-Cheby could shed light on building more efficient architectures for the area of graph learning.

\section{REFERENCES}

[1] Martın Abadi, Ashish Agarwal, Paul Barham, Eugene Brevdo, Zhifeng Chen, Craig Citro, Greg S Corrado, Andy Davis, Jeffrey Dean, Matthieu Devin, et al. 2015. TensorFlow: Large-scale machine learning on heterogeneous systems, 2015 Software available from tensorflow. org 1, 2 (2015).

[2] Song Bai, Feihu Zhang, and Philip H. S. Torr. 2019. Hypergraph Convolution and Hypergraph Attention. arXiv preprint arXiv:1901.08150 (2019). https://academic. microsoft.com/paper/2911251106

[3] Filippo Maria Bianchi, Daniele Grattarola, Lorenzo Livi, and Cesare Alippi 2019. Graph Neural Networks with convolutional ARMA filters. arXiv preprint arXiv:1901.01343 (2019). https://academic.microsoft.com/paper/2912636151

[4] Joan Bruna, Wojciech Zaremba, Arthur Szlam, and Yann Lecun. 2014. Spectral networks and locally connected networks on graphs. In International Conference on Learning Representations (ICLR2014), CBLS, April 2014. http-openreview.

[5] Heng Chang, Yu Rong, Tingyang Xu, Wenbing Huang, Honglei Zhang, Peng Cui, Wenwu Zhu, and Junzhou Huang. 2020. A restricted black-box adversarial framework towards attacking graph embedding models. In Proceedings of the AAAI Conference on Artificial Intelligence, Vol. 34. 3389-3396.

[6] Deli Chen, Yankai Lin, Wei Li, Peng Li, Jie Zhou, and Xu Sun. 2019. Measuring and Relieving the Over-smoothing Problem for Graph Neural Networks from the Topological View. arXiv preprint arXiv:1909.03211 (2019).

[7] Yunpeng Chen, Haoqi Fan, Bing Xu, Zhicheng Yan, Yannis Kalantidis, Marcus Rohrbach, Shuicheng Yan, and Jiashi Feng. 2019. Drop an Octave: Reducing Spatial Redundancy in Convolutional Neural Networks with Octave Convolution. arXiv preprint arXiv:1904.05049 (2019).

[8] Michaël Defferrard, Xavier Bresson, and Pierre Vandergheynst. 2016. Convolutional neural networks on graphs with fast localized spectral filtering. neural information processing systems (2016), 3844-3852.

[9] Chenhui Deng, Zhiqiang Zhao, Yongyu Wang, Zhiru Zhang, and Zhuo Feng. 2020. GraphZoom: A Multi-level Spectral Approach for Accurate and Scalable Graph Embedding. In International Conference on Learning Representations. https: //openreview.net/forum?id=r1lGO0EKDH

[10] Claire Donnat, Marinka Zitnik, David Hallac, and Jure Leskovec. 2018. Learning Structural Node Embeddings via Diffusion Wavelets. In Proceedings of the 24th ACM SIGKDD International Conference on Knowledge Discovery \& Data Mining. $1320-1329$
[11] Hongyang Gao and Shuiwang Ji. 2019. Graph representation learning via hard and channel-wise attention networks. In Proceedings of the 25th ACM SIGKDD International Conference on Knowledge Discovery \& Data Mining. 741-749.

[12] Xavier Glorot and Yoshua Bengio. 2010. Understanding the difficulty of training deep feedforward neural networks. In Proceedings of the thirteenth international conference on artificial intelligence and statistics. 249-256.

[13] Fangda Gu, Heng Chang, Wenwu Zhu, Somayeh Sojoudi, and Laurent El Ghaoui. 2020. Implicit graph neural networks. arXiv preprint arXiv:2009.06211 (2020).

[14] Chaoyu Guan, Ziwei Zhang, Haoyang Li, Heng Chang, Zeyang Zhang, Yijian Qin, Jiyan Jiang, Xin Wang, and Wenwu Zhu. 2021. AutoGL: A Library for Automated Graph Learning. arXiv preprint arXiv:2104.04987 (2021).

[15] William L. Hamilton, Zhitao Ying, and Jure Leskovec. 2017. Inductive Representation Learning on Large Graphs. neural information processing systems (2017), $1024-1034$.

[16] David K. Hammond, Pierre Vandergheynst, and Rémi Gribonval. 2011. Wavelets on graphs via spectral graph theory. Applied and Computational Harmonic Analysis 30, 2 (2011), 129-150.

[17] Ming Jin, Heng Chang, Wenwu Zhu, and Somayeh Sojoudi. 2019. Power up! robust graph convolutional network against evasion attacks based on graph powering. arXiv preprint arXiv:1905.10029 (2019).

[18] Diederik P Kingma and Jimmy Ba. 2014. Adam: A method for stochastic optimization. arXiv preprint arXiv:1412.6980 (2014).

[19] Thomas N. Kipf and Max Welling. 2017. Semi-Supervised Classification with Graph Convolutional Networks. international conference on learning representations (2017).

[20] Johannes Klicpera, Aleksandar Bojchevski, and Stephan Günnemann. 2019. Predict then Propagate: Graph Neural Networks meet Personalized PageRank. In International Conference on Learning Representations (ICLR).

[21] Yujia Li, Daniel Tarlow, Marc Brockschmidt, and Richard S. Zemel. 2016. Gated Graph Sequence Neural Networks.. In ICLR (Poster). https://academic.microsoft. com/paper/2950898568

[22] Renjie Liao, Zhizhen Zhao, Raquel Urtasun, and Richard S. Zemel. 2019. LanczosNet: Multi-Scale Deep Graph Convolutional Networks. In ICLR 2019 : 7th International Conference on Learning Representations.

[23] Sitao Luan, Mingde Zhao, Xiao-Wen Chang, and Doina Precup. 2019. Break the Ceiling: Stronger Multi-scale Deep Graph Convolutional Networks. In Advances in Neural Information Processing Systems 32. Curran Associates, Inc., 10945-10955.

[24] Laurens van der Maaten and Geoffrey Hinton. 2008. Visualizing data using t-SNE. fournal of machine learning research 9, Nov (2008), 2579-2605.

[25] Takanori Maehara. 2019. Revisiting Graph Neural Networks: All We Have is Low-Pass Filters. arXiv preprint arXiv:1905.09550 (2019).

[26] Julian McAuley, Christopher Targett, Qinfeng Shi, and Anton Van Den Hengel. 2015. Image-based recommendations on styles and substitutes. In Proceedings of the 38th International ACM SIGIR Conference on Research and Development in Information Retrieval. 43-52.

[27] Christopher Morris, Martin Ritzert, Matthias Fey, William Hamilton, Jan Eric Lenssen, Gaurav Rattan, and Martin Grohe. 2019. Weisfeiler and Leman Go Neural: Higher-order Graph Neural Networks. AAAI 2019: Thirty-Third AAAI Conference on Artificial Intelligence 33, 1 (2019), 4602-4609. https://academic. microsoft.com/paper/2962810718

[28] Bryan Perozzi, Rami Al-Rfou, and Steven Skiena. 2014. Deepwalk: Online learning of social representations. In Proceedings of the 20th ACM SIGKDD international conference on Knowledge discovery and data mining. ACM, 701-710.

[29] Prithviraj Sen, Galileo Mark Namata, Mustafa Bilgic, Lise Getoor, Brian Gallagher, and Tina Eliassi-Rad. 2008. Collective Classification in Network Data. Ai Magazine 29, 3 (2008), 93-106.

[30] Oleksandr Shchur, Maximilian Mumme, Aleksandar Bojchevski, and Stephan Günnemann. 2018. Pitfalls of graph neural network evaluation. arXiv preprint arXiv:1811.05868 (2018)

[31] David I Shuman, Sunil K. Narang, Pascal Frossard, Antonio Ortega, and Pierre Vandergheynst. 2013. The emerging field of signal processing on graphs: Extending high-dimensional data analysis to networks and other irregular domains. IEEE Signal Processing Magazine 30, 3 (2013), 83-98.

[32] Nitish Srivastava, Geoffrey Hinton, Alex Krizhevsky, Ilya Sutskever, and Ruslan Salakhutdinov. 2014. Dropout: a simple way to prevent neural networks from overfitting. The journal of machine learning research 15, 1 (2014), 1929-1958.

[33] Wim Sweldens. 1998. The lifting scheme: A construction of second generation wavelets. SIAM journal on mathematical analysis 29, 2 (1998), 511-546.

[34] Nicolas Tremblay and Pierre Borgnat. 2014. Graph wavelets for multiscale community mining. IEEE Transactions on Signal Processing 62, 20 (2014), 52275239.

[35] Petar Veličković, Guillem Cucurull, Arantxa Casanova, Adriana Romero, Pietro Liò, and Yoshua Bengio. 2018. Graph Attention Networks. In ICLR 2018 : International Conference on Learning Representations 2018.

[36] Guangtao Wang, Rex Ying, Jing Huang, and Jure Leskovec. 2020. Direct multi-hop attention based graph neural network. arXiv preprint arXiv:2009.14332 (2020).

[37] Xiao Wang, Houye Ji, Chuan Shi, Bai Wang, Yanfang Ye, Peng Cui, and Philip S Yu. 2019. Heterogeneous graph attention network. In The World Wide Web Conference. 
2022-2032.

[38] Felix Wu, Amauri H. Souza, Tianyi Zhang, Christopher Fifty, Tao Yu, and Kilian Q. Weinberger. 2019. Simplifying Graph Convolutional Networks. In ICML 2019 : Thirty-sixth International Conference on Machine Learning. 6861-6871.

[39] Zonghan Wu, Shirui Pan, Fengwen Chen, Guodong Long, Chengqi Zhang, and Philip S Yu. 2019. A comprehensive survey on graph neural networks. arXiv preprint arXiv:1901.00596 (2019).

[40] Bingbing Xu, Huawei Shen, Qi Cao, Yunqi Qiu, and Xueqi Cheng. 2019. Graph Wavelet Neural Network. international conference on learning representations (2019).

[41] Zhilin Yang, William W. Cohen, and Ruslan Salakhutdinov. 2016. Revisiting semi-supervised learning with graph embeddings. In ICML 2016. 40-48.

[42] Runhao Zeng, Wenbing Huang, Mingkui Tan, Yu Rong, Peilin Zhao, Junzhou Huang, and Chuang Gan. 2019. Graph Convolutional Networks for Temporal Action Localization. In Proceedings of the IEEE International Conference on Computer Vision. 7094-7103.

[43] Kai Zhang, Yaokang Zhu, Jun Wang, and Jie Zhang. 2019. Adaptive structural fingerprints for graph attention networks. In International Conference on Learning Representations.

[44] Ziwei Zhang, Peng Cui, and Wenwu Zhu. 2020. Deep learning on graphs: A survey. IEEE Transactions on Knowledge and Data Engineering (2020).

\section{A DATASETS}

Joining the practice of previous works, we mainly focus on five node classification benchmark datasets under semi-supervised setting with different graph size and feature type. (1) Three citation networks: Citeseer, Cora and Pubmed [29], which aims to classify the research topics of papers. (2) A coauthor network: Coauthor CS which aims to predict the most active fields of study for each author from the KDD Cup 2016 challenge $^{1}$. (3) A co-purchase network: Amazon Photo [26] which aims to predict the category of products from Amazon. For the citation networks, we follow the public split setting provided by [41], that is, 20 labeled nodes per class in each dataset for training and $500 / 1000$ labeled samples for validation / test respectively. For the other two datasets, we follow the splitting setting from $[6,30]$. Statistical overview of all datasets is given in Table 3. Label rate denotes the ratio of labeled nodes fetched in training process.

Table 3: The overview of dataset statistics.

\begin{tabular}{lrrrrr}
\hline Dataset & Nodes & Edges & Classes & Features & Label rate \\
\hline Citeseer & 3,327 & 4,732 & 6 & 3,703 & 0.036 \\
Cora & 2,708 & 5,429 & 7 & 1,433 & 0.052 \\
Pubmed & 19,717 & 44,338 & 3 & 500 & 0.003 \\
Coauthor CS & 18,333 & 81,894 & 15 & 6,805 & 0.016 \\
Amazon Photo & 7,487 & 11,9043 & 8 & 745 & 0.021 \\
\hline
\end{tabular}

\section{B FULL ABLATION STUDIES}

\section{B.1 The Learned Attention on Low- and High-frequency Components}

In this Section, we also show how the learned attentions of SpGAT w.r.t the best proportion for Citeseer, Cora and Pubmed which are demonstrated in Table 4. Interestingly, despite the small proportion, the attention weight of low-frequency components learned by SpGAT is much larger than that of high-frequency components in each layer consistently. Hence, SpGAT is successfully to capture the importance of low- and high-frequency components of graphs in the spectral domain. Moreover, as pointed out by [10, 25], the low-frequency components in graphs usually indicate smooth varying signals which can reflect the locality property in graphs. It implies that the local structural information is important for these datasets. This may explain why GAT also gains good performance on these datasets.

\footnotetext{
${ }^{1}$ https://kddcup2016.azurewebsites.net
}

Table 4: Learned attention weights $\alpha_{L}$ and $\alpha_{H}$ of SpGAT for low- and high-frequency w.r.t the best proportion of low frequency components $d$ (number followed after the name of datasets).

\begin{tabular}{lcc|cc|cc}
\hline Dataset & \multicolumn{3}{c|}{ Citeseer (15\%) } & \multicolumn{2}{c|}{ Cora (5\%) } & \multicolumn{2}{c}{ Pubmed (10\%) } \\
\hline Attention filter weights & $\alpha_{L}$ & $\alpha_{H}$ & $\alpha_{L}$ & $\alpha_{H}$ & $\alpha_{L}$ & $\alpha_{H}$ \\
\hline Learned value (first layer) & $\mathbf{0 . 8 4}$ & 0.16 & $\mathbf{0 . 7 2}$ & 0.23 & $\mathbf{0 . 8 6}$ & 0.14 \\
\hline Learned value (second layer) & $\mathbf{0 . 9 4}$ & 0.06 & $\mathbf{0 . 9 3}$ & 0.07 & $\mathbf{0 . 9 3}$ & 0.07 \\
\hline
\end{tabular}

Table 5: The results of ablation study on low- and highfrequency components.

\begin{tabular}{lccc}
\hline Methods & Citeseer (15\%) & Cora (5\%) & Pubmed (10\%) \\
\hline with low-frequency & 57.7 & 66.8 & 76.7 \\
\hline with high-frequency & 70.9 & 82.4 & 80.4 \\
\hline SpGAT & $\mathbf{7 2 . 3}$ & $\mathbf{8 3 . 8}$ & $\mathbf{8 0 . 8}$ \\
\hline
\end{tabular}

\section{B.2 Only Low- and High-frequency Components}

To further elaborate the importance of low- and high-frequency components in SpGAT, we conduct the ablation study on the classification results by testing only with low- or high-frequency components w.r.t the best proportion. Specially, we manually set $\alpha_{L}$ or $\alpha_{H}$ to 0 during testing stage to observe how the learned low- and high-frequency components in graphs affect the classification accuracy. From Table 5, we can observe that:

- Both low- and high-frequency components are essential for the model. Since removing any components downgrade the over performance.

- SpGAT with very small proportion (5\% - 15\%) of low-frequency components can achieve the comparable results to those obtained by full SpGAT. It reads that the low-frequency components contain more information that can contribute to the feature representation learned from the model.

\section{B.3 t-SNE Visualization of Learned Embeddings}

To evaluate the effectiveness of the learned features of SpGAT qualitatively, we also depict the t-SNE visualization [24] of learned embeddings of SpGAT on three citation datasets in Figure 5. The representation exhibits discernible clustering in the projected 2D space. In Figure 5, the color indicates the class label in each dataset. Compared with the other methods, the intersections of different classes in SpGAT are more separated. It verifies the discriminative power of SpGAT across the classes.

\section{RELATED WORKS}

Spectral convolutional networks on graphs. Existing methods of defining a convolutional operation on graphs can be broadly divided into two categories: spectral based and spatial based methods [14, 44]. We focus on the spectral graph convolutions in this paper. Spectral CNN [4] first attempts to generalize CNNs to graphs based on the spectrum of the graph Laplacian and defines the convolutional kernel in the spectral domain. ChebyNet [8] introduces a fast localized convolutional filter on graphs via Chebyshev polynomial approximation. Vanilla GCN [19] further extends the spectral graph convolutions considering networks of significantly larger scale by several simplifications. Lanczos algorithm is utilized in LanczosNet [22] to 


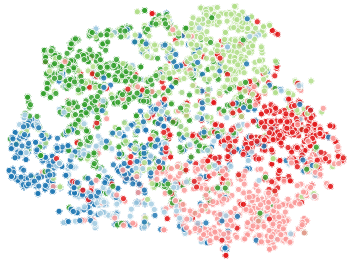

(a) VanillaGCN on Citeseer

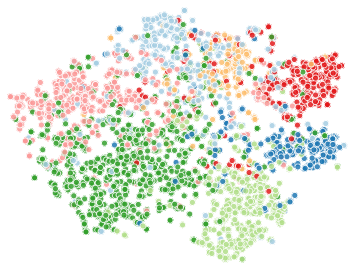

(e) VanillaGCN on Cora

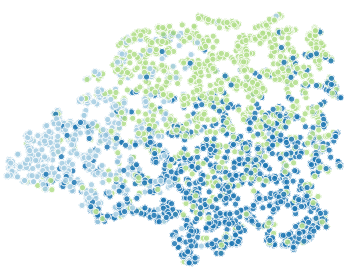

(i) VanillaGCN on Pubmed

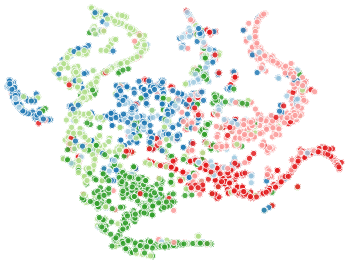

(b) GWNN on Citeseer

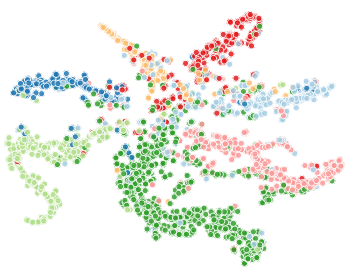

(f) GWNN on Cora

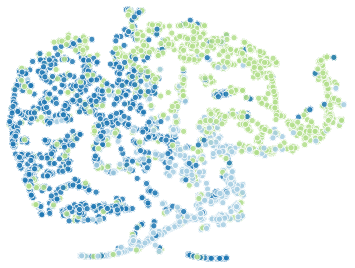

(j) GWNN on Pubmed

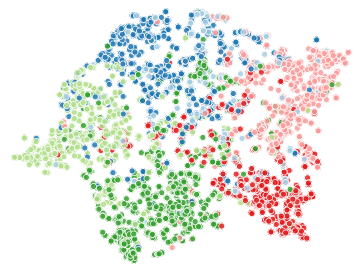

(c) GAT on Citeseer

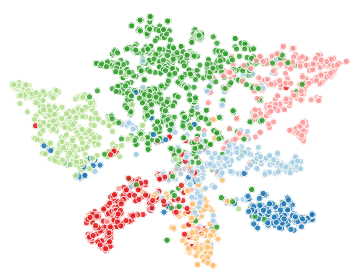

(g) GAT on Cora

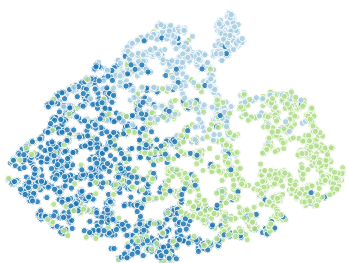

(k) GAT on Pubmed

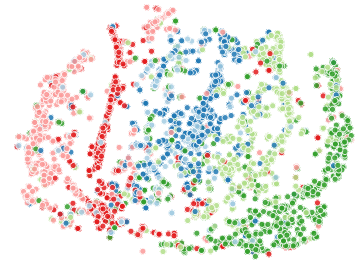

(d) SpGAT on Citeseer

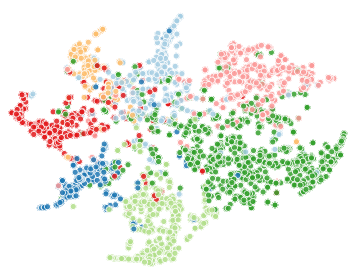

(h) SpGAT on Cora

Figure 5: The t-SNE visualization of SpGAT comparing with other baselines on citation datasets. Each color corresponds to a different class that the embeddings belongs to.

construct low-rank approximations of the graph Laplacian for convolution. [40] first attempts to construct graph neural networks with graph wavelets. SGC [38] further reduces the complexity of Vanilla GCN by successively removing the non-linearities between consecutive layers. [23] then generalizes the spectral graph convolution in block Krylov subspace forms to make use of multi-scale information. Despite their effective performance, all these convolution theorem based methods lack the strategy to explicitly treat low- and high-frequency components with different importance.

Space/spectrum-aware feature representation. In computer vision, [7] first defines space-aware feature representations based on scale-space theory and reduces spatial redundancy of vanilla $\mathrm{CNN}$ models by proposing the Octave Convolution (OctConv) model. To our knowledge, this is the first time that spectrum-aware feature representations are considered in irregular graph domain and established with graph convolutional neural networks.

Spectral Graph Wavelets Theoretically, the lifting scheme is proposed for the construction of wavelets that can be adapted to irregular graphs in [33]. [16] defines wavelet transforms appropriate for graphs and describes a fast algorithm for computation via fast Chebyshev polynomial approximation. For applications, [34] utilizes graph wavelets for multi-scale community mining and obtains a local view of the graph from each node. [10] introduces the property of graph wavelets that describes information diffusion and learns structural node embeddings accordingly. [40] first attempts to construct graph neural networks with graph wavelets. These works emphasize the local and sparse property of graph wavelets for Graph Signal Processing both theoretically and practically. 